\title{
Protective Effect of Zuojin Fang on Lung Injury Induced by Sepsis through Downregulating the JAK1/STAT3 Signaling Pathway
}

\author{
Jiangning Yin $\mathbb{D}^{1,2}$ Zhan Yu, ${ }^{1}$ Chuanyong Hou, ${ }^{1}$ Yigen Peng, ${ }^{1}$ Jianpeng Xiao, ${ }^{2}$ \\ and Jun Jiang $\mathbb{D}^{2}$ \\ ${ }^{1}$ Emergency Department, The Affiliated Jiangning Hospital of Nanjing Medical University, Nanjing, Jiangsu 211100, China \\ ${ }^{2}$ School of Pharmacy, Jiangsu University, Xuefu Road, Zhenjiang, Jiangsu 212013, China \\ Correspondence should be addressed to Jiangning Yin; jsyinjn@163.com and Jun Jiang; jiangjuntcm2007@hotmail.com
}

Received 6 August 2020; Revised 5 December 2020; Accepted 16 December 2020; Published 7 January 2021

Academic Editor: Li-kang SUN

Copyright (C) 2021 Jiangning Yin et al. This is an open access article distributed under the Creative Commons Attribution License, which permits unrestricted use, distribution, and reproduction in any medium, provided the original work is properly cited.

\begin{abstract}
Lung injury was the common and serious complication of sepsis, a systemic inflammatory response syndrome caused by severe infections. Chinese medicine had unique advantages in attenuating inflammatory response, such as Zuojinfang (ZJF). ZJF was a classical compound herb formula composed of Coptidis Rhizoma and Euodiae Fructus in a ratio of $6: 1$. In this paper, 15 ingredients in ZJF were identified and 8 of them absorbed into rat's serum were quantified by HPLC-MS/MS. Subsequently, sepsis-induced lung injury model was replicated in rats by cecal ligation and puncture. $60 \mathrm{SD}$ rats were randomly divided into 6 groups $(n=10)$ : control group (CON), sham group (Sham), model group (MOD), ZJF low-dose group (ZJF-L), ZJF high-dose group (ZJF-H), and prednisolone group (PNSL). Within the next $24 \mathrm{~h}$, the levels of inflammatory factors, correlation between active ingredients and inflammatory cytokines, the pathological changes of lung tissue, and protein expression of the JAK1/STAT3 signaling pathways were analyzed one by one. Finally, the concentration order of components absorbed in rat serum was berberine $>$ palmatine $>$ jatrorrhizine $>$ coptisine $>$ evodin $>$ chlorogenic acid $>$ evodiamine. Compared with the MOD group, the TNF- $\alpha$, IL- 6 , and IFN- $\gamma$ in the ZJF-H group were significantly reduced $(p<0.05)$. Moreover, the TNF- $\alpha$ decreased significantly accompanied by the increase of berberine, chlorogenic acid, jatrorrhizine, palmatine, evodin, and evodiamine in serum (negative correlation, $p<0.05$ ). Compared with the MOD, the area of lung injury, the expressions of JAK1, p-JAK1, STAT3, and p-STAT3 were significantly decreased under the treatment of ZJF $(p<0.05)$. Therefore, downregulating the JAK1/STAT3 signaling pathways was a potential avenue of ZJF in reversing lung injury induced by sepsis.
\end{abstract}

\section{Introduction}

Sepsis was defined as a life-threatening organ dysfunction caused by a dysregulated host response to infection [1]. At present, sepsis was a leading cause of death and disability in intensive care units (ICU). The incidence of sepsis was between 66 and 300 per 100000 people and showed an increasing trend [2-4]. Mortality estimates for sepsis ranged from 27 to $36 \%$ in developed countries [5]. In its preliminary stage, sepsis tended to induce lung injury, characterized by damaged alveolar capillary barrier, edema, pulmonary infiltrates, and hypoxemia [6]. Nowadays, treatment of sepsisinduced lung injury includes antibiotic [7], glucocorticoids
[8], corticosteroid [9], ketamine [10], simvastatin [11], and artesunate [12]. However, the use of antibiotics inevitably leads to the emergence of resistant bacteria, a series of adverse reactions $[13,14]$. Other drugs were also accompanied by some troubling side effects in osteoporosis and glaucoma [15], psychiatric adverse effect [16], cardiovascular diseases [17], liver and muscle injury [18], and cytotoxicity [19], respectively.

Fortunately, it was found that some Chinese herbs had unique advantages in reducing the production of drugresistant bacteria and inhibiting the generation of proinflammatory factors. Moreover, active ingredients obtained from Chinese herbs or compound preparation of Chinese medicine like emodin [20], ginsenoside $\mathrm{Rg}_{1}$ [21], 
glycyrrhizic acid [22], and Xuebijing injection [23] usually provided beneficial effects in anti-infective, anti-inflammatory, and immunomodulatory.

Zuojinfang (ZJF) was a classical formula composed of Coptidis Rhizoma (CR) and Euodiae Fructus (EF) in a ratio of $6: 1$. ZJF was always applied to treat gastric ulcer, gastroesophageal reflux, gastritis, and pyloric obstruction [24, 25]. The active ingredients of CR are alkaloids, including berberine, jatrorrhizine, palmatine, and coptisine; especially, berberine is the most abundant compound [26]. Studies showed that CR extracts and berberine can effectively improve the survival rate of rats with sepsis [27]. Furthermore, Huang-Lian-Jie-Du Decoction has been widely used in the treatment of sepsis by activating the choline pathway and inhibiting the HMGB-1/TLR4/NF- $\kappa \mathrm{B}$ signaling pathway [28]. In addition, relevant studies have proved that evodiamine has a therapeutic effect on inflammation by reducing the expression of IL- $1 \beta$, IL- 6 , and TNF- $\alpha$ [29]. Based on these researches, it was believed that ZJF has a unique pharmacological action in reversing sepsisinduced lung injury.

The purpose of this study was to verify the efficacy and mechanism of ZJF in reversing lung injury induced by sepsis. Firstly, HPLC-MS/MS was applied for the qualitative and quantitative analysis of ZJF. And then the 8 ingredients absorbed into the rat's serum were also determined by HPLC-MS/MS. The inflammatory factor levels were tested by enzyme-linked immunosorbent assay (ELISA). Pathologic changes in lung tissues were evaluated via pathological section with hematoxylin and eosin (HE) staining. The expressions of JAK, p-JAK1, STAT3, and p-STAT3 were determined by western blot assay. Our study not only evaluated the therapeutic effect of ZJF on lung injury induced by sepsis but also provided a new perspective for resolving the mechanism of ZJF in regulating the JAK1/STAT3 signaling pathway.

\section{Materials and Method}

2.1. Ethics Statement. Animal experiments were carried out in accordance with the Guidelines of Animal Experimentation in Jiangsu University (Zhenjiang, China), and the protocol was also approved by its Animal Ethics Committee.

2.2. Reference Materials and Medicinal Materials. Chlorogenic acid, jatrorrhizine, palmatine, berberine, evodin, evodiamine, and rutaecarpine (purity $>98.0 \%$ ) were purchased from the National Institute for the Control of Pharmaceutical and Biological Products (Beijing, China). Coptisine (purity $>98.5 \%$ ) was purchased from Prof. Hao Zhang (West China School of Pharmacy, Sichuan University, Chengdu, China). CR was provided by Nanjing Pharmacy (Nanjing, China). EF was provided by Evodiae Planting Base (Hubei, China). CR and EF were identified by Prof. Chengying Wu (Jiangsu Provincial Academy of Chinese Medicine, Nanjing, China) as Coptidis Rhizoma Franch and Evodiarutaecar pa (Juss.) Benth. var. officinalis (Dode) Huang, respectively. ZJF was obtained by mixing CR (120 g) and EF (20 g), crushing, and passing through 60 molybdenum sieves.
2.3. Model Establishment and Grouping. Cecal ligation and puncture [30-33] was applied to establish sepsis model in rats. 60 healthy male SD rats (SPF, $220 \pm 20 \mathrm{~g}$ ) were provided by the Animal Center of Jiangsu University. All rats were randomly divided into 6 groups $(n=10)$ : control group (CON, normal saline, $10 \mathrm{~mL} / \mathrm{kg}$ ), sham operation group (Sham, normal saline, $10 \mathrm{~mL} / \mathrm{kg}$ ), model group (MOD, normal saline, $10 \mathrm{~mL} / \mathrm{kg}$ ), ZJF low-dose group (ZJF-L, $20 \mathrm{mg} / \mathrm{kg} /$ day), ZJF high-dose group (ZJF-H, $40 \mathrm{mg} / \mathrm{kg} /$ day), and positive control prednisolone group (PNSL, $10 \mathrm{mg} / \mathrm{kg} / \mathrm{day}$ ).

\subsection{Preparation of Standard and Sample Solutions}

2.4.1. Standard Solutions. Mixed standard stock solution containing chlorogenic acid $(34.5 \mu \mathrm{g} / \mathrm{mL})$, jatrorrhizine $(35.0 \mu \mathrm{g} / \mathrm{mL}), \quad$ coptisine $\quad(18.0 \mu \mathrm{g} / \mathrm{mL})$, palmatine $(13.0 \mu \mathrm{g} / \mathrm{mL})$, berberine $(43.0 \mu \mathrm{g} / \mathrm{mL})$, evodin $(35.0 \mu \mathrm{g} / \mathrm{mL})$, evodiamine $(42.0 \mu \mathrm{g} / \mathrm{mL})$, and rutaecarpine $(32.0 \mu \mathrm{g} / \mathrm{mL})$ was prepared in methanol. The solutions were stored in refrigerator at $4^{\circ} \mathrm{C}$ and kept in darkness. The mixed standard stock solution was diluted into a series of standard working solution by methanol.

2.4.2. Preparation of Extract Samples. ZJF (CR $120 \mathrm{~g}$ and EF $20 \mathrm{~g}$ ) was extracted twice with $65 \%$ ethanol by refluxing for $90 \mathrm{~min}$ each time [34]. Two-part extraction was incorporated, evaporated under vacuum, and lyophilized to obtain the ZJF extracts. Freeze-dried powder $(0.1 \mathrm{~g})$ was weighed into a $50 \mathrm{~mL}$ polypropylene centrifugal tube, and $50 \mathrm{~mL}$ of methanol was added for sonication about $40 \mathrm{~min}$. Before analysis by HPLC, the supernatant solution was filtered through a $0.45 \mu \mathrm{m}$ pore size Millipore filter, and then a total volume of $10 \mu \mathrm{L}$ was injected.

2.4.3. Preparation of Serum Samples. After intragastric administration of ZJF extracts (freeze-dried powder dispersed in $0.5 \% \mathrm{CMC}-\mathrm{Na}$ ), the rats were subjected to blood collection from orbit at $6 \mathrm{~h}, 12 \mathrm{~h}$, and $24 \mathrm{~h}$, respectively. $0.2 \mathrm{~mL}$ of plasma was taken into the heparinized centrifuge tube, centrifuged at $4000 \mathrm{rpm}$ for $10 \mathrm{~min}$, and $50 \mu \mathrm{L}$ of supernatant was taken and frozen-spare.

$0.5 \mathrm{~mL}$ of ethyl acetate was added to $50 \mu \mathrm{L}$ of the serum, vortexed for $2 \mathrm{~min}$, and centrifuged for $10 \mathrm{~min}$ to obtain $400 \mu \mathrm{L}$ of the supernatant. It was vacuum-dried at $40^{\circ} \mathrm{C}$ until ethyl acetate was evaporated, reconstituted by adding $100 \mu \mathrm{L}$ of methanol, ultrasonically mixed for $5 \mathrm{~min}$, and centrifuged for $10 \mathrm{~min}$ to obtain $80 \mu \mathrm{L}$ of the supernatant for chromatographic analysis.

2.5. Chromatographic and Mass Spectrometry Conditions. ZORBAX XDC- $\mathrm{C}_{18}$ column $(4.6 \mathrm{~mm} \times 50 \mathrm{~mm}, 1.8 \mu \mathrm{m})$ was used for the chromatographic separation under gradient elution with acetonitrile $-0.3 \%$ glacial acetic acid (HCN\%, 0$7 \mathrm{~min}: 5-8$; $7-13 \mathrm{~min}: \quad 8-11 \mathrm{~min} ; 13-25 \mathrm{~min}: 11-25$; $25-$ $45 \mathrm{~min}: 25-32$; $45-50 \mathrm{~min}: 30$; 50-75 min: 30-60; flow rate: $0.4 \mathrm{~mL} / \mathrm{min}$ ) at a flow rate $0.4 \mathrm{~mL} / \mathrm{min}$. The column injection volume was $5 \mu \mathrm{L}$, and column temperature was $20^{\circ} \mathrm{C}$. MS acquisition was carried out in the ESI-positive ionization mode. Detection mode was set to multiple reaction monitoring (MRM, Table 1). The conditions were set as follows: 
TABLE 1: Screening parameters of the compounds in ZJF by HPLCMS/MS.

\begin{tabular}{lccc}
\hline Compounds & $t_{\mathrm{R}}{ }^{\mathrm{a}}(\mathrm{min})$ & $\mathrm{m} / \mathrm{z}[\mathrm{M}]^{+}$ & $\mathrm{m} / \mathrm{z}[\mathrm{M}+\mathrm{H}]^{+}$ \\
\hline 1 & 20.87 & & 355.3 \\
2 & 24.60 & & 342.6 \\
3 & 27.74 & & 340.5 \\
4 & 28.76 & 424.0 & \\
5 & 31.56 & $322.2^{\mathrm{b}}$ & \\
6 & 34.27 & 338.3 & \\
7 & 34.95 & 336.2 & \\
8 & 35.12 & 338.3 & \\
9 & 36.90 & 302.4 & \\
10 & 38.18 & 320.2 & \\
11 & 40.47 & 352.3 & \\
12 & 44.71 & 336.2 & \\
13 & 65.66 & 471.8 & \\
14 & 68.55 & & 304.4 \\
15 & 70.58 & & 288.4 \\
\hline
\end{tabular}

${ }^{\mathrm{a}}$ Times of retention. ${ }^{\mathrm{b}}[\mathrm{M}-\mathrm{HCHO}]^{+}$.

corona discharge current $1.16 \mu \mathrm{A}$, capillary voltage $4000 \mathrm{~V}$, a nitrogen flow rate $10 \mathrm{~L} / \mathrm{min}$, gas temperature $350^{\circ} \mathrm{C}$, scan range $100-600(\mathrm{~m} / \mathrm{z})$, and nebulizer pressure $25 \mathrm{psi}$.

\subsection{Method Validation of HPLC-MS/MS}

2.6.1. Regression Equations, Linear Range, and LOD of 8 Components. The stock solutions of eight standards were prepared and diluted to six appropriate concentrations for establishing the calibration curves of HPLC-MS/MS (Figure 1). The regression equations were achieved after linear regression of the peak areas versus the corresponding concentrations. The LOQ for each analyte under the chromatographic conditions was determined at the $\mathrm{S} / \mathrm{N}$ of 3 and 10, respectively. The results indicated that all eight reference compounds had a good linearity $\left(r^{2}>0.9997\right)$ in a relatively wide concentration range (Table 2 ).

2.6.2. Precision, Repeatability, Stability, and Recovery. Intraday and interday variations were chosen to determine the precision of the developed assay. Three different concentrations of standards were prepared. The intraday variation was determined by analyzing the six replicates within a single day. Interday variation was examined in six consecutive days. Repeatability was confirmed with solutions prepared from $\mathrm{ZJF}$, and it was injected into the apparatus at $0,2,4,8,12$, 16 , and $24 \mathrm{~h}$, respectively. Variations were expressed by RSD. It indicated that the intraday, interday, repeatability, and stability of RSD values of the eight compounds were all less than $3.0 \%$.

$10 \mu \mathrm{L}$ mixed standard solution (10 ppm) was precisely removed into $0.1 \mathrm{~mL}$ blank plasma samples and their peak areas were recorded $(A)$ by HPLC-MS/MS after the same pretreatment as described in Section 2.4. Another $10 \mu \mathrm{L}$ mixed standard solution (10 ppm) was added into $0.1 \mathrm{~mL}$ methanol with the same operation and their areas were measured as " $B$." Their recovery was calculated by " $(A / B) \times 100 \%$ ". The recovery rate of 8 active ingredients in serum showed stable recovery between $83.7 \%$ and $89.5 \%$.

2.7. Detection of TNF- $\alpha, I F N-\gamma, I L-6$, and SOD in Serum. Detection was performed according to the manufacturer's instructions for the enzyme-linked immunosorbent assay kits. $50 \mu \mathrm{L}$ standard solution of series concentrations and $10 \mu \mathrm{L}$ sample solution were prepared; then, the diluent was added $(40 \mu \mathrm{L})$. Standard and sample wells were added with horseradish peroxidase- (HRP-) labelled antibody $(100 \mu \mathrm{L})$ to detect the antibody. The reaction wells were sealed with the sealing plate membrane and kept in $37^{\circ} \mathrm{C}$ water bath for $60 \mathrm{~min}$. Discard the liquid, dry the plates, fill them up with washing fluid for $1 \mathrm{~min}$, shake off the washing liquid, dry the plates, and repeat the washing process 5 times. The substrates were added $(50 \mu \mathrm{L})$ to all the wells and incubated for $15 \mathrm{~min}$ at $37^{\circ} \mathrm{C}$. $15 \mathrm{~min}$ later, the OD value of each well was measured at $450 \mathrm{~nm}$ wavelength. The standard curve was obtained with the linear regression of standard concentration and OD value, and the corresponding concentration of the OD value of the sample was found on this curve. The concentration of samples could be found from the standard curve according to their OD values.

2.8. HE Staining. Collected lung tissues were fixed in $10 \%$ formalin for $48 \mathrm{~h}$. The right upper lobe and hilar organizations were routinely washed, dehydrated, embedded in paraffin, and cut into $5 \mu \mathrm{m}$ serial sections. Then, the sections were gradually dewaxed, dehydrated, stained with HE, immersed in xylene and alcohol, stained with hematoxylin for $5 \mathrm{~min}$, stained with eosin for $3 \mathrm{~min}$, and reimmersed in alcohol and xylene. Finally, the morphological changes $[35,36]$ of tissues were observed under an optical microscope (Olympus IX71/IX81, Olympus Corporation, Japan).

2.9. Expression of JAK1, p-JAK1, STAT3, and p-STAT3 in Lung Tissue. Protein samples of lung tissue were prepared and subjected to protein gel electrophoresis (Bio-Rad). The gel was transferred to a membrane, blocked, and incubated with a primary antibody: the JAK1 (Santa Cruz, CA, USA; sc-376996), p-JAK1 (Santa Cruz, CA, USA; sc-377043), STAT3 (Santa Cruz, CA, USA; sc-482), and p-STAT3 (Santa Cruz, CA, USA; sc-8001-R) diluted 1:1000. The diluted primary antibody was incubated at room temperature for $2 \mathrm{~h}$. Secondary antibody was incubated for 30-60 min. Membranes were visualized with enhanced chemiluminescence. ImageJ software (National Institutes of Health, Bethesda, MD) was used for analyzing protein bands. The relative density of each band was calculated to $\beta$-actin as the control.

2.10. Statistical Analysis. All data were presented as the mean \pm SD. Statistical analysis was performed using GraphPad Prism 5 (GraphPad software, USA). Differences were analyzed by one-way analysis of variance (Tukey/compare all pairs of columns). Differences $p<0.05$ were considered as significant. 


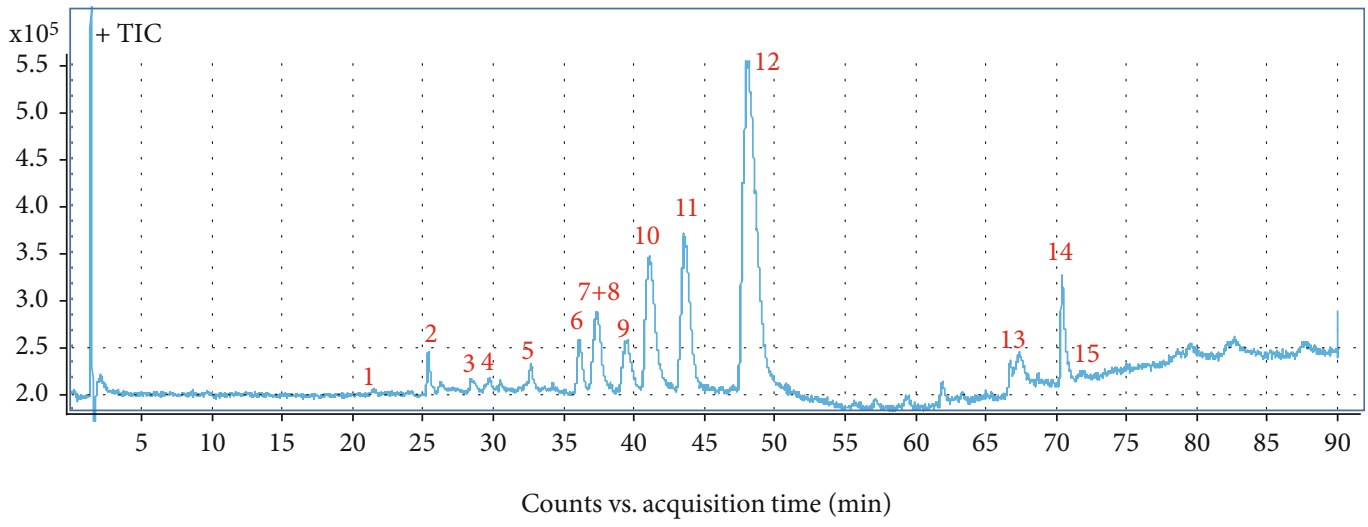

(a)

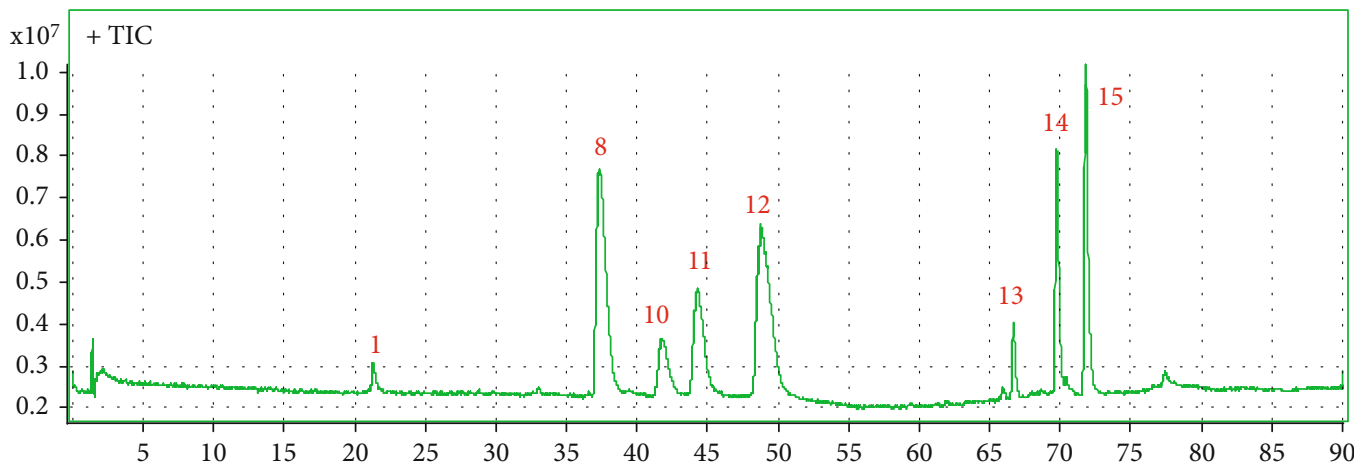

(b)

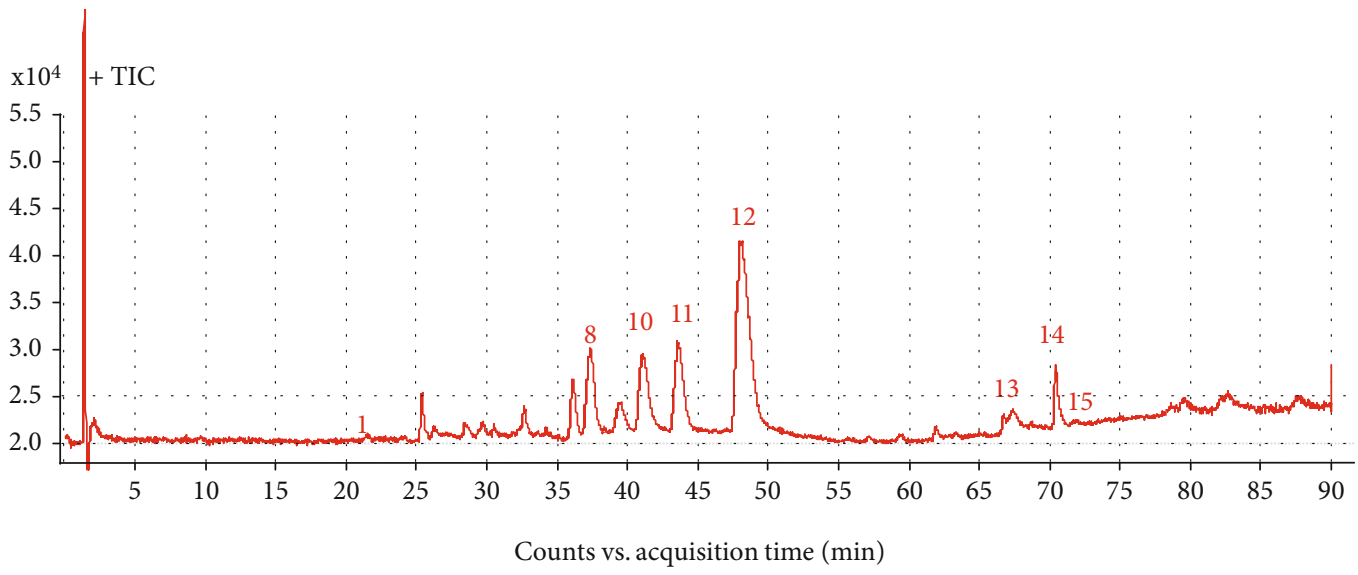

(c)

Figure 1: Typical total ion chromatogram (TIC) of 15 active ingredients in ZJF extracts. (a) ZJF sample. (b) Standard solution. (c) Serum sample. Peaks: 1: chlorogenic acid; 8: jatrorrhizine; 10: coptisine; 11: palmatine; 12: berberine; 13: evodin; 14: evodiamine; 15: rutaecarpine.

\section{Results}

3.1. Profiling Chemical Composition in ZJF. The extracted ion chromatogram (EIC) of 15 components (Figure 2) was retrieved in ZJF according to retention time $\left(t_{\mathrm{R}}\right)$ and corresponding parent ion $(\mathrm{m} / \mathrm{z})$ produced by HPLC-MS/MS (Table 1). They were chlorogenic acid (1), dehydroevodiamine (2), evocarpine (3), taraxerone (4), berberastine (5), columbamine (6), epiberberine (7), jatrorrhizine (8), dehydroevodiamine (9), coptisine (10), palmatine (11), berberine (12), evodin (13), evodiamine (14), and rutaecarpine (15).
Based on the external standard method, the contents of compounds 1,8 , and $10-15$ were $0.64 \pm 0.14 \mathrm{mg} / \mathrm{g}, 13.15$ $\pm 2.64 \mathrm{mg} / \mathrm{g}, \quad 38.16 \pm 4.51 \mathrm{mg} / \mathrm{g}, \quad 14.97 \pm 2.89 \mathrm{mg} / \mathrm{g}, \quad 79.06$ $\pm 8.35 \mathrm{mg} / \mathrm{g}, 2.52 \pm 0.56 \mathrm{mg} / \mathrm{g}, 0.56 \pm 0.12 \mathrm{mg} / \mathrm{g}$, and 0.35 $\pm 0.08 \mathrm{mg} / \mathrm{g}$, respectively. The content of berberine was the highest and rutaecarpine was the lowest in ZJF extracts (Supplementary Materials).

3.2. Dynamic Changes of Ingredients Absorbed into Serum. The contents of the above 8 components in serum at $6 \mathrm{~h}$, $12 \mathrm{~h}$, and $24 \mathrm{~h}$ after oral administration of ZJF at high and 
TABLE 2: Regression equations, correlation coefficient, linear range, LOD, and recovery of 8 components by HPLC-MS/MS.

\begin{tabular}{|c|c|c|c|c|c|}
\hline Compounds & Regression equation & $r^{2}$ & Linear range $(\mathrm{ng} / \mathrm{mL})$ & LOQ (ng/mL) & Recovery (\%) \\
\hline 1 & $Y=5053.5 X-282.5$ & 0.9999 & $0.5-50.0$ & 0.5 & 85.4 \\
\hline 8 & $Y=1048721 X+101447$ & 0.9999 & $0.5-50.0$ & 0.5 & 87.2 \\
\hline 10 & $Y=258201 X-40.1$ & 0.9999 & $0.5-50.0$ & 0.5 & 83.7 \\
\hline 11 & $Y=508402 X-28108$ & 0.9999 & $0.5-50.0$ & 0.5 & 88.1 \\
\hline 12 & $Y=1271407 X-111917$ & 0.9997 & $2.0-150.0$ & 2.0 & 86.8 \\
\hline 13 & $Y=32151 X-3568.5$ & 0.9999 & $0.5-50.0$ & 0.5 & 86.3 \\
\hline 14 & $Y=211093 X-688.8$ & 0.9998 & $0.1-10.0$ & 0.1 & 88.2 \\
\hline 15 & $Y=838756 X-77674$ & 0.9998 & $0.1-10.0$ & 0.1 & 89.5 \\
\hline
\end{tabular}

Note: $Y$ is the peak area; $X$ is the concentration injected. LOQ refers to the limit of quantification. 1: chlorogenic acid; 8: jatrorrhizine; 10: coptisine; 11: palmatine; 12: berberine; 13: evodin; 14: evodiamine; 15: rutaecarpine.

low doses were quantitatively analyzed. Finally, only the rutaecarpine was not detected, and the other 7 components were successfully quantified. Results showed that the content of Coptis alkaloids in serum was always the highest, including berberine $(100.28 \pm 2.31 \mathrm{ng} / \mathrm{mL})$, jatrorrhizine $(11.42 \pm 0.71 \mathrm{ng} / \mathrm{mL})$, coptisine $(9.33 \pm 0.43 \mathrm{ng} / \mathrm{mL})$, and palmatine $(20.73 \pm 1.55 \mathrm{ng} / \mathrm{mL})$, especially the berberine. Besides, the contents of evodin $(2.64 \pm 0.44 \mathrm{ng} / \mathrm{mL})$ and chlorogenic acid $(3.85 \pm 0.78 \mathrm{ng} / \mathrm{mL})$ obtained from Euodiae Fructus were also prominent in serum. Furthermore, the content of these active ingredients in serum decreased significantly from $6 \mathrm{~h}$ to $24 \mathrm{~h}$ after administration (Table 3 ).

\subsection{Dynamic Changes of Inflammation-Related Factors.} TNF- $\alpha$, IFN- $\gamma$, and IL- 6 were the inflammatory factors, and SOD was an antioxidant index, which were related to inflammation. Compared with the sham group, the levels of TNF- $\alpha$, IFN- $\gamma$, and IL- 6 at $6 \mathrm{~h}, 12 \mathrm{~h}$, and $24 \mathrm{~h}$ in the MOD group were all significantly increased $(p<0.05)$ along with the significant decrease of SOD $(p<0.05)$. After the intervention of ZJF and PNSL, the expressions of TNF- $\alpha$, IFN- $\gamma$, and IL- 6 were reduced and the SOD level was increased (Figure 3). More specifically, compared with the MOD group, the expressions of TNF- $\alpha$, IL- 6 , and IFN- $\gamma$ in ZJF-H group were significantly reduced $(p<0.05)$ and the level of SOD was significantly increased $(p<0.01)$. Therefore, ZJF significantly inhibited the inflammatory expression in septic rats.

3.4. Correlation Analysis. The correlation analysis was conducted between the components and the inflammatory factors (TNF- $\alpha$, IL-6, and IFN- $\gamma$ ) at same time points $(6 \mathrm{~h}, 12 \mathrm{~h}$, and $24 \mathrm{~h})$. The levels of inflammatory cytokines were regarded as the ordinate, and the contents of active ingredients were the abscissa for linear regression (Figure 4). The results showed that the TNF- $\alpha$ was negatively correlated with berberine $(p<0.0001)$, chlorogenic acid $(p<0.01)$, jatrorrhizine $(p<0.01)$, palmatine $(p<0.01)$, evodin $(p<0.05)$, and evodiamine $(p<0.05)$. Moreover, there was no significant correlation between the other inflammatory factors (IL-6 and IFN- $\gamma$ ) and the active ingredients.
3.5. Pathological Changes in Lung Tissue. In the CON or Sham groups, the alveolar wall was thin; no exudate was found in the alveolar cavity and interstitium under the microscope. However, in the MOD group, dark red plaques or spot-like lesions of different sizes were observed on the lung surface accompanied by swelling and hyperemia of lung tissue. In the ZJF-L group, alveolar interstitium was hyperemia and edema, neutrophils were moderately infiltrated, capillaries were dilated, and some alveolar spaces were widened. In the ZJF-H group, alveolar interstitium was slightly congested and edematous with mild neutrophil infiltration, telangiectasia, and hyperemia (Figure 5(a)). Pathological score showed that the damage area in the MOD group was significantly increased compared with the sham group $(p<0.01)$. After treatment with ZJF and PNSL, the area of lung injury decreased significantly compared with the MOD ( $p<0.05$, Figure 5(b)).

3.6. Expression of JAK1, p-JAK1, STAT3, and p-STAT3 in Lung Tissue. Compared with the sham group, the protein expressions of JAK1, p-JAK1, STAT3, and p-STAT3 in the MOD were increased $(p<0.05)$. Compared with the MOD, the PNSL also significantly downregulated the protein expression of JAK1, p-JAK1, STAT3, and p-STAT3 $(p<0.01)$. Similar to the PNSL group, the expression of JAK1, p-JAK1, STAT3, and p-STAT3 was also downregulated in the ZJF-H group $(p<0.01)$. Therefore, ZJF reversed sepsis caused lung injury by downregulating the expression of JAK1/STAT3-related proteins (Figure 6).

\section{Discussion}

According to our previous studies, the contents of the $8 \mathrm{com}$ ponents in ZJF extract were in the order of berberine $>$ coptisine $>$ palmatine $>$ jatrorrhizine $>$ evodin $>$ chlorogenic acid $>$ evodiamine $>$ rutaecarpine. After oral administration of ZJF, the concentration of components absorbed into the blood is in this order berberine $>$ palmatine $>$ jatrorrhizine $>$ coptisine $>$ evodin $>$ chlorogenic acid $>$ evodiamine. Therefore, the contents of berberine, palmatine, jatrorrhizine, coptisine, evodin, chlorogenic acid, and evodiamine in both the extract and the serum were prominent. However, in 

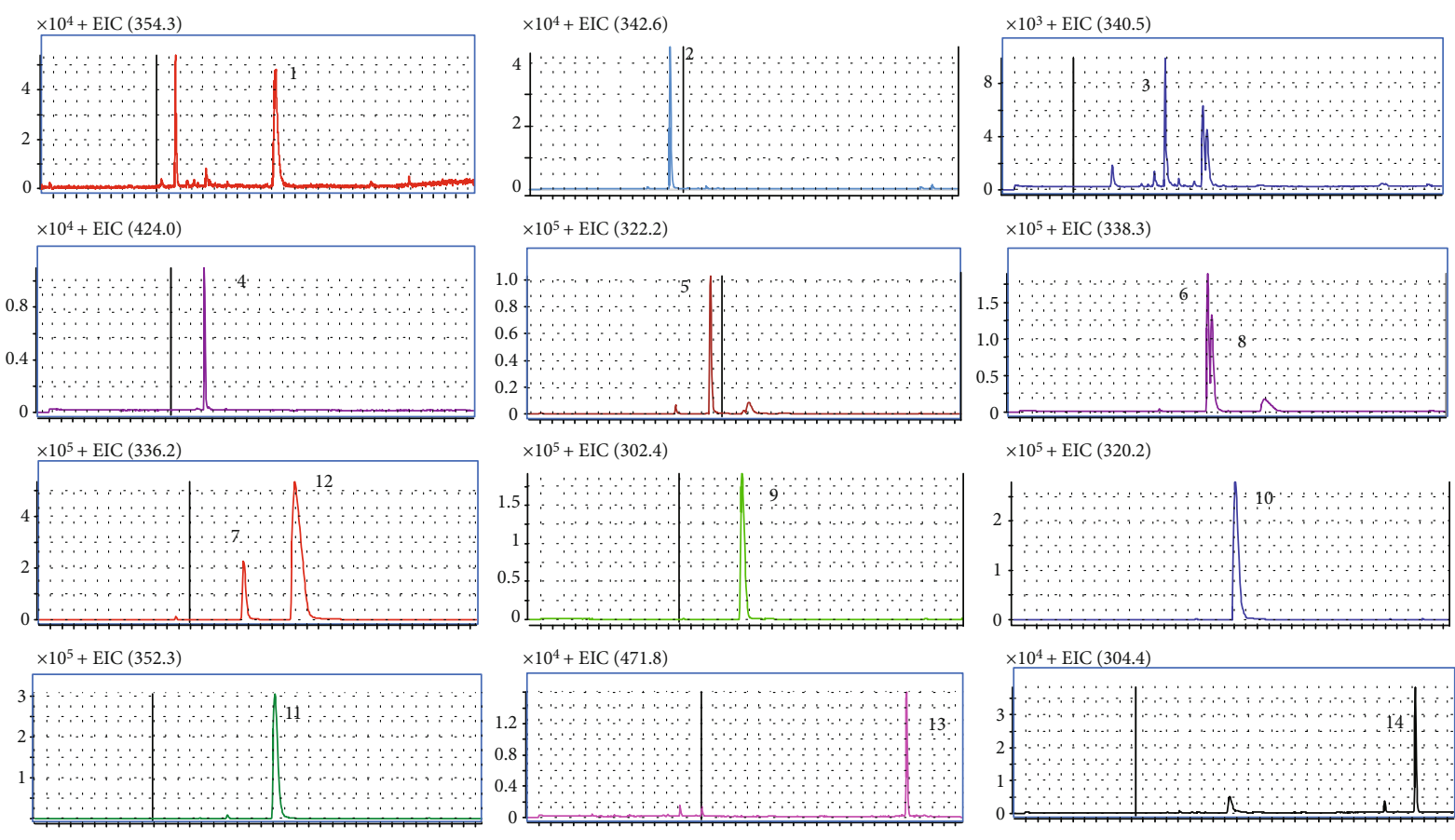

$\times 10^{5}+\operatorname{EIC}(320.2)$

$\times 10^{4}+\operatorname{EIC}(288.4)$

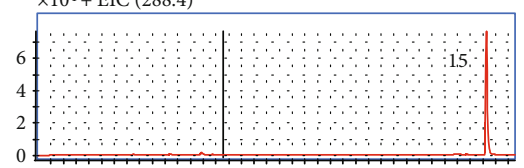

$\times 10^{4}+\operatorname{EIC}(471.8)$
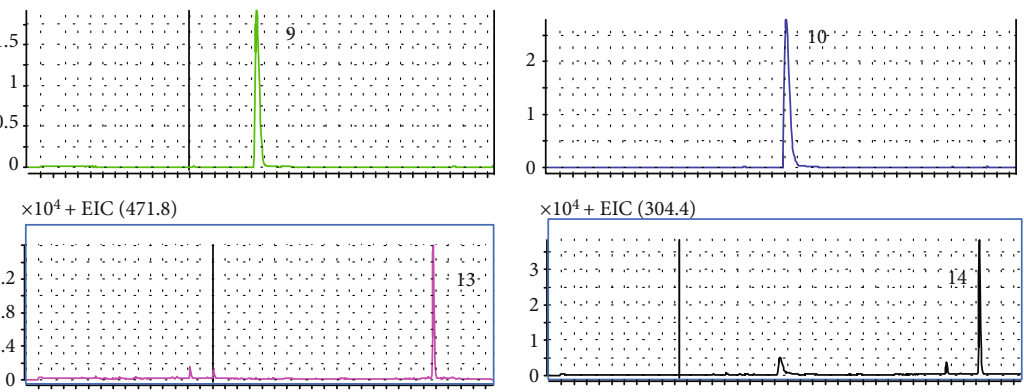

(a)

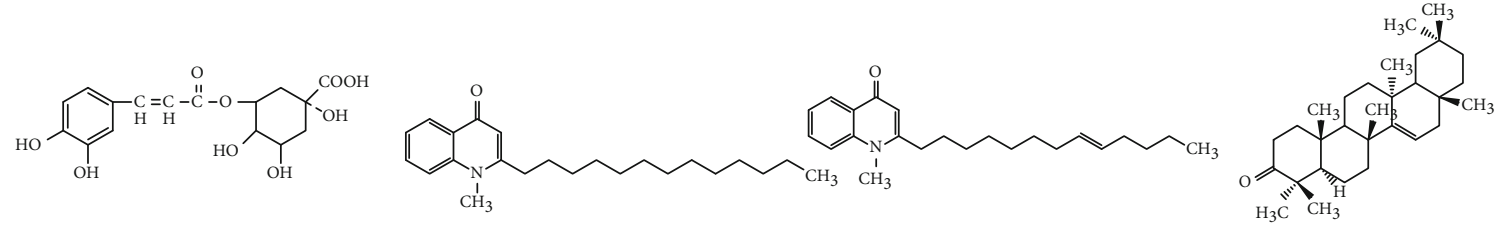

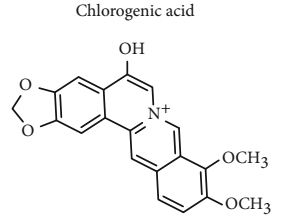

Berberastine<smiles>C[N+]1=C2c3[nH]c4ccccc4c3C=CN2C(=O)C2C=CC=CC21</smiles>

Dehydroevodiamine

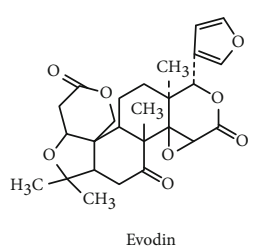

Dihydroevocarpine<smiles>COc1cc2cc[n+]3cc4c(OC)c(OC)ccc4cc3c2cc1O</smiles>

Columbamine

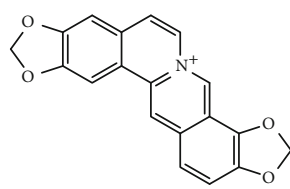

Coptisine

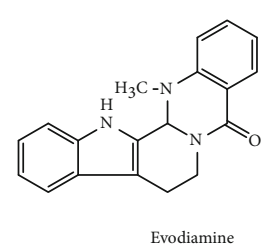

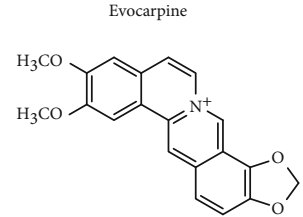

Epiberberine

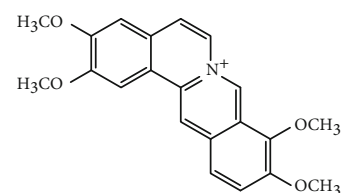

Palmatine

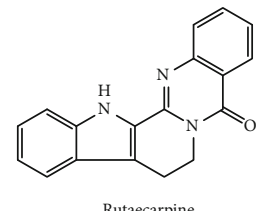

Rutaecarpine

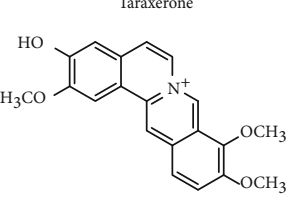

Jatrorrhizine

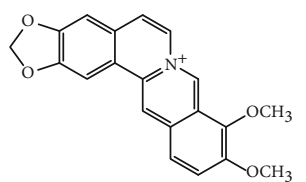

Berberine

(b)

FIGURE 2: Qualitative analysis of active components in ZJF. (a) Extracting ion chromatogram (EIC) of 15 components in ZJF extract. (b) Chemical structures of identified 15 components in ZJF extract. 
TABLE 3: Concentration changes of ZJF active ingredients in rat serum after different treatment time $(n=3)$.

\begin{tabular}{lcccccccccc}
\hline \multirow{2}{*}{ Dose } & $P_{\mathrm{t}}$ & $\begin{array}{c}\text { Chlorogenic } \\
\text { acid }\end{array}$ & Jatrorrhizine & Coptisine & Palmatine & Berberine & Evodin & Evodiamine Rutaecarpine \\
& & $6 \mathrm{~h}$ & $1.96 \pm 0.17$ & $4.70 \pm 0.46$ & $3.75 \pm 0.63$ & $9.65 \pm 0.82$ & $59.95 \pm 3.10$ & $7.25 \pm 0.75$ & $0.99 \pm 0.12$ & $\mathrm{nd}$ \\
ZJF-L & $12 \mathrm{~h}$ & $1.08 \pm 0.19$ & $2.09 \pm 0.22$ & $3.15 \pm 0.37$ & $3.10 \pm 0.62$ & $11.09 \pm 1.17$ & $2.86 \pm 0.58$ & $0.33 \pm 0.06$ & $\mathrm{nd}$ & $\mathrm{nd}$ \\
$(20 \mathrm{mg} / \mathrm{kg})$ & $24 \mathrm{~h}$ & $1.13 \pm 0.26$ & $3.03 \pm 0.46$ & $1.78 \pm 0.53$ & $3.04 \pm 0.62$ & $3.87 \pm 0.77$ & $2.76 \pm 0.47$ & $0.15 \pm 0.04$ & $\mathrm{nd}$ \\
& $6 \mathrm{~h}$ & $3.85 \pm 0.78$ & $11.42 \pm 0.71$ & $9.33 \pm 0.43$ & $20.73 \pm 1.55$ & $100.28 \pm 2.31$ & $14.75 \pm 1.26$ & $2.64 \pm 0.44$ & $\mathrm{nd}$ & $\mathrm{nd}$ \\
ZJF-H & $12 \mathrm{~h}$ & $2.56 \pm 0.81$ & $4.04 \pm 0.50$ & $7.64 \pm 0.60$ & $6.50 \pm 0.79$ & $21.32 \pm 1.89$ & $6.74 \pm 0.33$ & $0.52 \pm 0.07$ & $\mathrm{nd}$ \\
$(40 \mathrm{mg} / \mathrm{kg})$ & $24 \mathrm{~h}$ & $2.74 \pm 0.62$ & $6.26 \pm 0.95$ & $3.97 \pm 0.41$ & $5.62 \pm 0.94$ & $6.30 \pm 0.60$ & $6.25 \pm 0.94$ & $0.23 \pm 0.05$ & $\mathrm{nd}$ \\
\hline
\end{tabular}
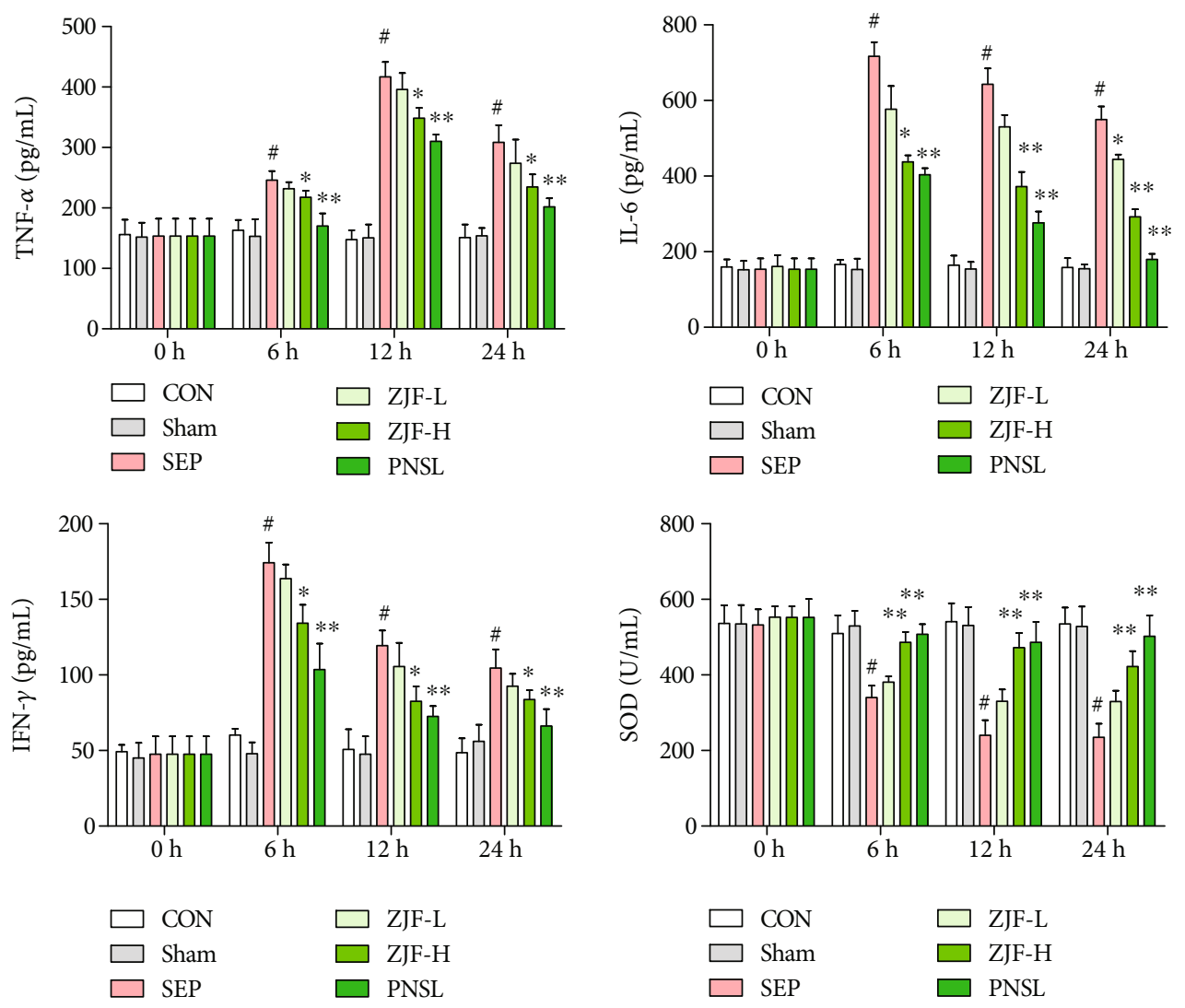

FIGURE 3: Dynamic changes of inflammation-related factors in rat serum. CON: control group; Sham: sham operation group; SEP: model group; ZJF-L: Zuojinfang low-dose group; ZJF-H: Zuojinfang high-dose group; PNSL: prednisolone; TNF- $\alpha$ : tumor necrosis factor $\alpha$; IFN$\gamma$ : interferon $\gamma$; IL-6: interleukin 6; SOD: superoxide dismutase. ${ }^{*} p<0.05$ and ${ }^{* *} p<0.01$ compared with the SEP group. ${ }^{*} p<0.05$ compared with the sham group.

Chinese Pharmacopoeia, berberine was also utilized as the only indicator for the quality control of ZJF. Simultaneous determination of multicomponents established in this study provided an important technical support for the quality control of ZJF and the subsequent investigation of integrative mechanism.

Undoubtedly, excessive inflammation response was the major contributing factor for sepsis-induced organ injury [37]. This immune dysregulation was a complex interplay of diverse signaling pathways which were aberrantly activated or suppressed losing their normal regulated functions. Excessive expression of inflammatory mediators in sepsis was associated with multiple signaling pathways, such as Janus kinase (JAK)/signal transducer and activator of transcription (STAT). The JAK/STAT signaling pathway leads to the accumulation of a large number of proinflammatory cytokines and increases the inflammatory response. In recent years, the significance of JAK1/STAT3 signaling involved in regulating inflammation and injury of sepsis attracted more attention due to its simple and effective activation. Sepsis induced JAK1 phosphorylation, followed by STAT3 phosphorylation, which activated the JAK1/STAT3 signaling pathway. Subsequently, the inflammatory response was increased under the trigger of p-STAT3, thereby increasing 

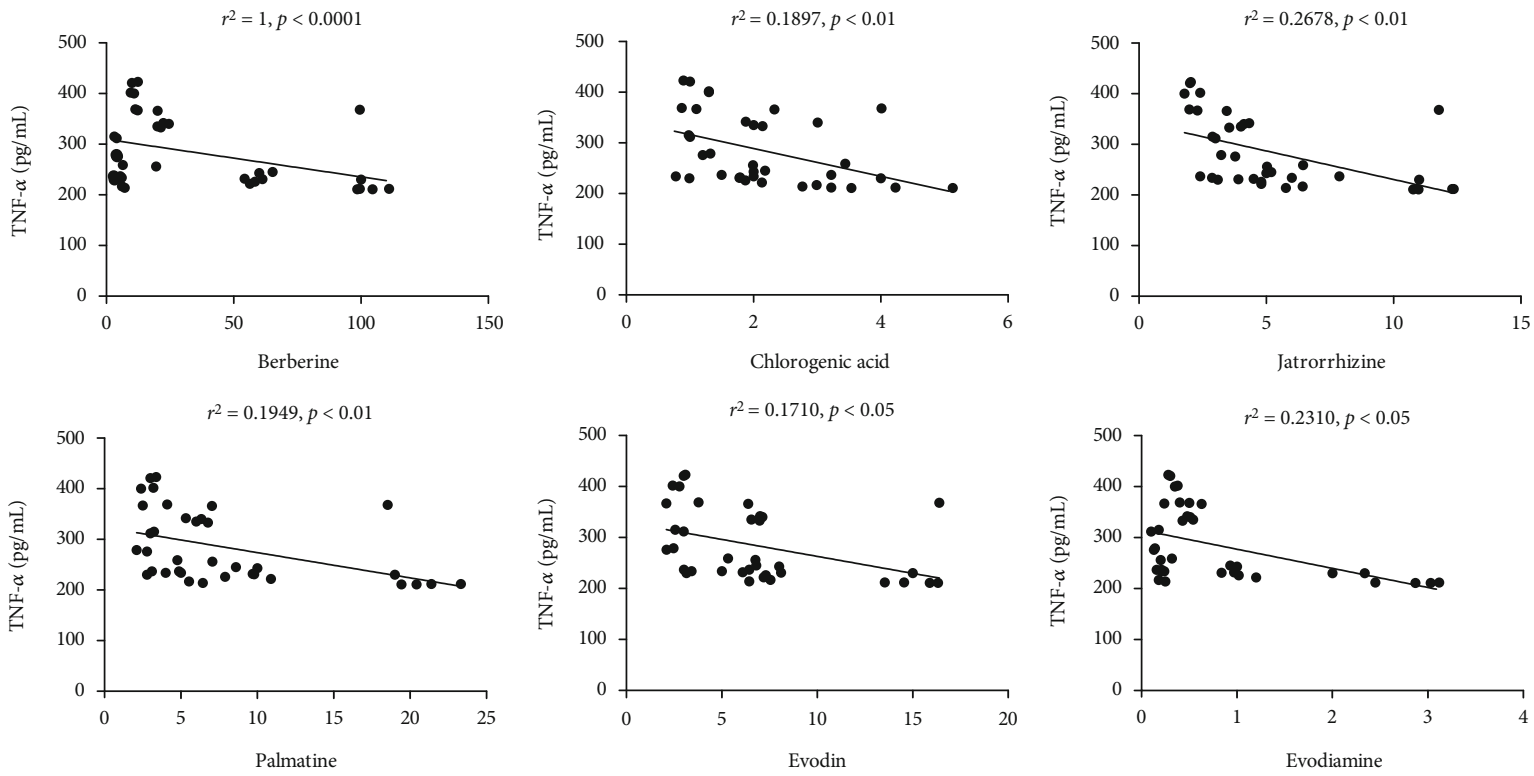

FIGURE 4: Correlation analysis between active components and the inflammatory factors in serum. There was a negative correlation between 6 active components (chlorogenic acid, jatrorrhizine, palmatine, berberine, evodiamine, and rutaecarpine) and TNF- $\alpha(p<0.05)$.

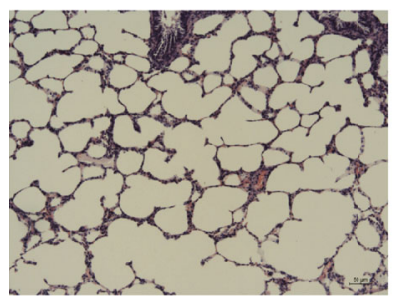

CON

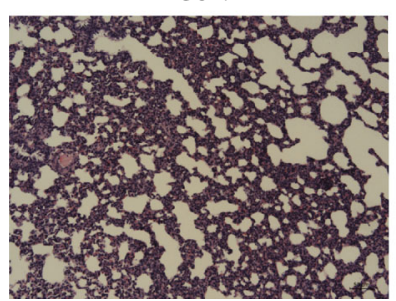

ZJF-L

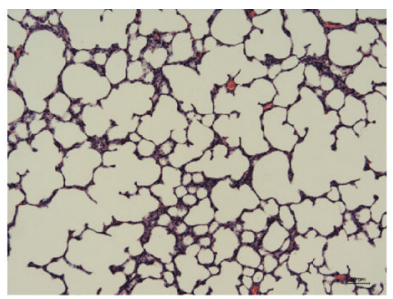

Sham

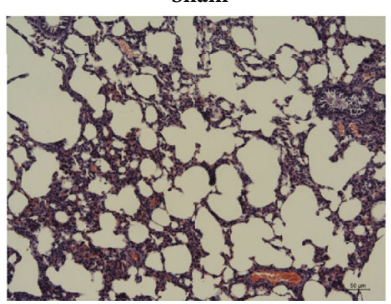

ZJF-H

(a)

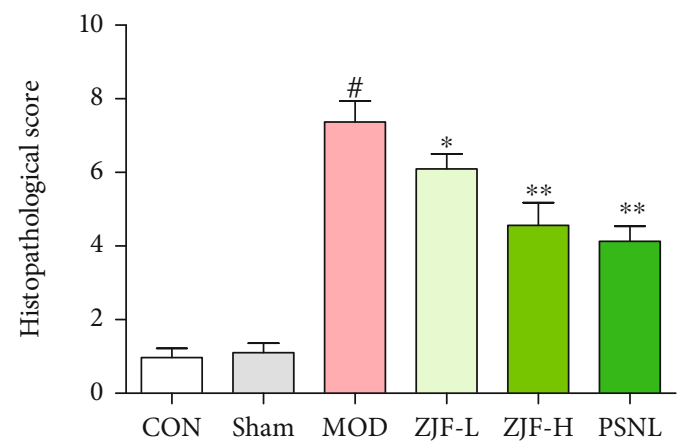

(b)

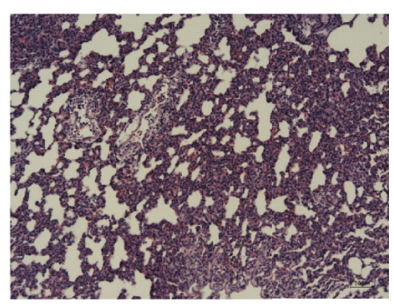

MOD

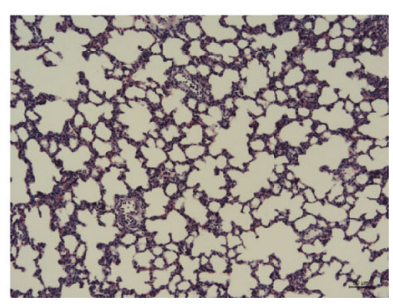

PNSL

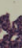

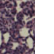

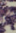




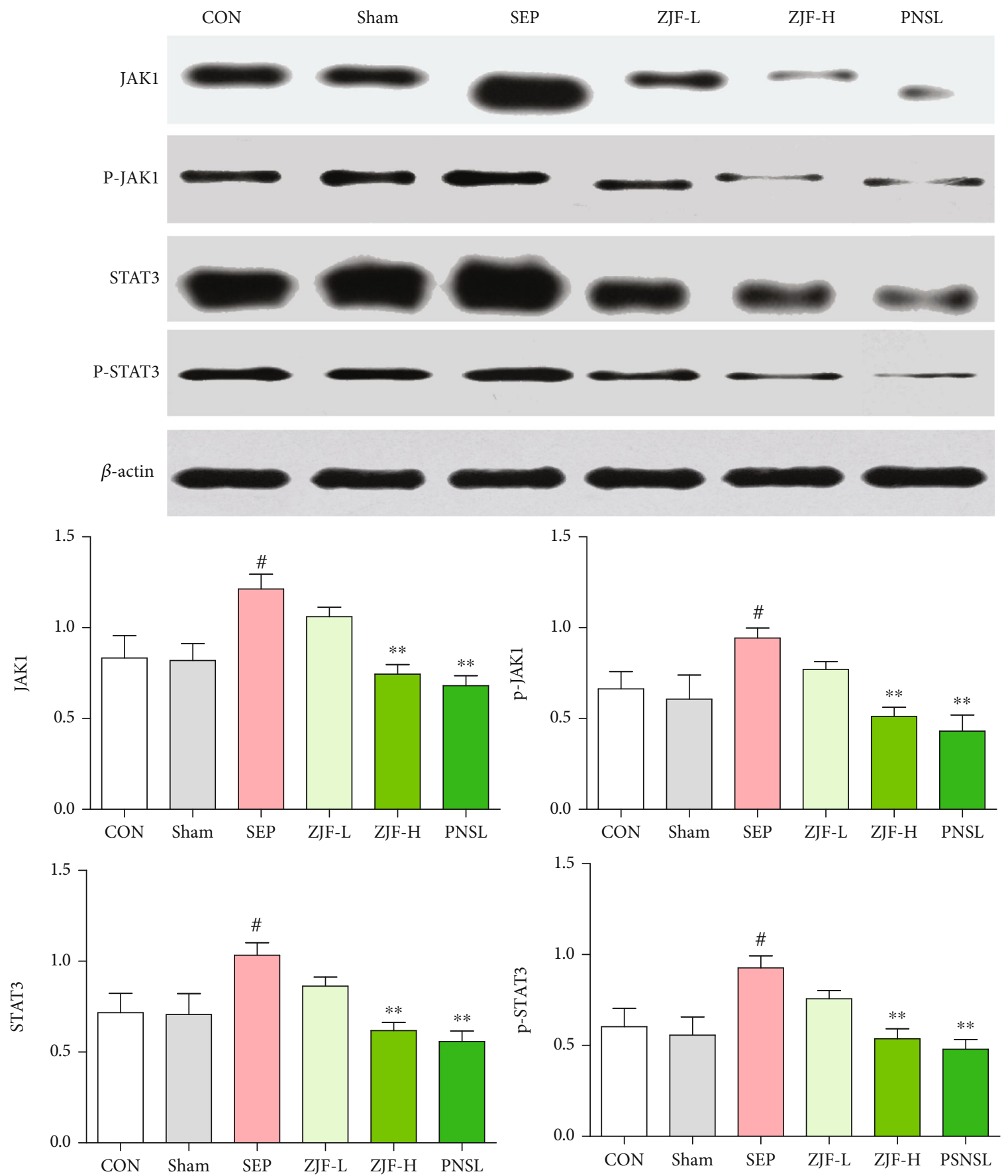

FIGURE 6: Expression of JAK1, p-JAK1, STAT3, and p-STAT3 proteins in rat lung tissue. CON: control group; Sham: sham operation group; SEP: model group; ZJF-L: Zuojinfang low-dose group; ZJF-H: Zuojinfang high-dose group; PNSL: prednisolone; JAK1: Janus kinase 1; STAT3: signal transducer and activator of transcription. ${ }^{* *} p<0.01$ compared with the SEP group. ${ }^{*} p<0.05$ compared with the sham group.

the severity of lung injury [38]. It was showed that the continued deterioration of sepsis is associated with excessive expression of inflammatory factors caused by the JAK1STAT3 signaling pathway. Similarly, when the JAK1STAT3 signaling pathway was inhibited, it reduced the severe inflammatory response caused by sepsis. After oral administration of ZJF, the expressions of JAK1, p-JAK1, STAT3, and p-STAT3 proteins were significantly reduced $(p<0.05)$ and ultimately reduced the level of inflammatory mediators $(p<0.05)$. Therefore, this study confirmed that ZJF could reverse sepsis-induced lung injury by inhibiting the JAK1STAT3 signal pathway and reducing the expression of inflammatory factors.

In order to explore the intrinsic communication of components and inflammation, the correlations between 8 components and 3 inflammatory factors (TNF- $\alpha$, IFN- $\gamma$, and IL-6) in serum were analyzed according to the data collected at $6 \mathrm{~h}, 12 \mathrm{~h}$, and $24 \mathrm{~h}$ after oral administration of ZJF extract. Fortunately, the concentration of TNF- $\alpha$ decreased significantly accompanied by the increase of various components, 
including berberine $(p<0.0001)$, chlorogenic acid $(p<0.01)$, jatrorrhizine $(p<0.01)$, palmatine $(p<0.01)$, evodin $(p<0.05)$, and evodiamine $(p<0.05)$. However, this notable negative correlation did not exist in other inflammatory factors and ingredients. This study suggested that berberine, chlorogenic acid, jarrorhizine, palmatine, evodin, and evodiamine had inhibitory effects on sepsis-induced inflammatory response, especially the TNF- $\alpha$. Through literature review, other laboratory also found that berberine [39] and chlorogenic acid [40, 41] could inhibit the expression of inflammatory factors through the JAK/STAT signaling pathway.

There are few studies on antisepsis of ZJF, Coptis chinensis (CR), Evodia rutaecarpa (EF), and their main components (berberine and rutaecarpine). In the existing reports, it was found that ZJF, CR, and EF downregulated the expression of inflammatory factors, such as TNF- $\alpha$. The main mechanism was related to the NF- $\kappa \mathrm{B}$ signaling pathway $[42,43]$. However, the expression of inflammatory cytokines (TNF- $\alpha$, IL-6, IL- $1 \beta$, and so on) can also be regulated through other signaling pathways, such as JAK-STAT [44-46]. Therefore, the goal of this paper was to prove that ZJF, CR, and EF exerted antisepsis effect by inhibiting the JAK-STAT signaling pathway to downregulate the expression of inflammatory factors. Fortunately, it was found that there was a negative correlation between serum concentrations of multiple components and TNF- $\alpha$ levels. Therefore, downregulating the level of inflammatory factors by inhibiting the JAK-STAT signaling pathway was one of the mechanisms of ZJF for the treatment of sepsis. ZJF reversed the lung injury induced by sepsis was related to the inhibition of inflammatory response under the synergistic effect of multicomponents, such as chlorogenic acid, jatrorrhizine, palmatine, berberine, evodiamine, and rutaecarpine. In the subsequent study, the mechanism of these components in treating lung injury induced by sepsis will be continued to explore.

\section{Conclusions}

ZJF reversed the lung injury caused by sepsis through inhibiting JAK1/STAT3 expression and subsequently reducing inflammatory mediator production. More importantly, berberine, chlorogenic acid, jarrorhizine, palmatine, evodin, and evodiamine were important potential substances of ZJF in treating lung injury induced by sepsis.

\section{Data Availability}

The data used to support the findings of this study are available from the corresponding author upon request.

\section{Conflicts of Interest}

All authors had no conflicts of interest regarding the publication of this study.

\section{Acknowledgments}

This work was supported by the National Natural Science Foundation of China (no. 81703773) and the Natural Science Foundation of Jiangsu Province (no. BK20170560).

\section{Supplementary Materials}

Table 1: content of 8 active components in ZJF extract $(\mathrm{mg} / \mathrm{g}, n=3)$. Figure 1: typical HPLC-DAD chromatograms of the standard solution and ZJW extract. (Supplementary Materials)

\section{References}

[1] M. Singer, C. S. Deutschman, C. W. Seymour et al., "The third international consensus definitions for sepsis and septic shock (sepsis-3)," Journal of the American Medical Association, vol. 315, no. 8, pp. 801-810, 2016.

[2] E. K. Stevenson, A. R. Rubenstein, G. T. Radin, R. S. Wiener, and A. J. Walkey, "Two decades of mortality trends among patients with severe sepsis: a comparative meta-analysis," Critical Care Medicine, vol. 42, no. 3, pp. 625-631, 2014.

[3] X. Lv and H. Wang, "Pathophysiology of sepsis-induced myocardial dysfunction," Military Medicine Research, vol. 3, no. 1, pp. 30-63, 2016.

[4] E. Damiani, A. Donati, G. Serafini et al., "Effect of performance improvement programs on compliance with sepsis bundles and mortality: a systematic review and meta-analysis of observational studies," PLoS One, vol. 10, no. 5, article e0125827, 2015.

[5] A. Keeley, P. Hine, and E. Nsutebu, "The recognition and management of sepsis and septic shock: a guide for non-intensivists," Postgraduate Medicine Journal, vol. 93, no. 1104, pp. 626-634, 2017.

[6] Y. Z. Li, J. H. Chen, C. F. Tsai, and W. L. Yeh, "Anti-inflammatory property of imperatorin on alveolar macrophages and inflammatory lung injury," Lloydia, vol. 82 , no. 4, pp. 10021008, 2019.

[7] R. Sherwin, M. E. Winters, G. M. Vilke, and G. Wardi, "Does early and appropriate antibiotic administration improve mortality in emergency department patients with severe sepsis or septic shock?," Journal of Emergency Medicine, vol. 53, no. 4, pp. 588-595, 2017.

[8] E. K. Incerpi, L. M. Oliveira, E. M. Pereira, and R. Soncini, "Inhibition of endogenous glucocorticoid synthesis aggravates lung injury triggered by septic shock in rats," International Journal of Experimental Pathology, vol. 96, no. 3, pp. 133139,2015

[9] B. Long and A. Koyfman, "Controversies in corticosteroid use for sepsis," Journal of Emergency Medicine, vol. 53, no. 5, pp. 653-661, 2017.

[10] K. Li, J. Yang, and X. Han, "Ketamine attenuates sepsisinduced acute lung injury via regulation of HMGB1-RAGE pathways," International Immunopharmacology, vol. 34, no. 1, pp. 114-128, 2016.

[11] Y. Wang, W. Yang, X. Zhao, and R. Zhang, "Experimental study of the protective effect of simvastatin on lung injury in rats with sepsis," Inflammation, vol. 41, no. 1, pp. 104-113, 2018. 
[12] T. H. Cao, S. G. Jin, D. S. Fei et al., "Artesunate protects against sepsis-induced lung injury via heme oxygenase-1 modulation," Inflammation, vol. 39, no. 2, pp. 651-662, 2016.

[13] C. Torres-Barcelo, "The disparate effects of bacteriophages on antibiotic-resistant bacteria," Emerging Microbes and Infections, vol. 7, no. 1, pp. 1-12, 2018.

[14] D. I. Andersson and D. Hughes, "Selection and transmission of antibiotic-resistant bacteria," Microbiology Spectrum, vol. 5, no. 4, 2017.

[15] D. R. Overby and A. F. Clark, "Animal models of glucocorticoid-induced glaucoma," Experimental Eye Research, vol. 141, no. 1, pp. 15-22, 2015.

[16] L. B. Drozdowicz and J. M. Bostwick, "Psychiatric adverse effects of pediatric corticosteroid use," Mayo Clinic Proceedings, vol. 89, no. 6, pp. 817-834, 2014.

[17] B. Short, J. Fong, V. Galvez, W. Shelker, and C. K. Loo, "Sideeffects associated with ketamine use in depression: a systematic review," The Lancet Psychiatry, vol. 5, no. 1, pp. 65-78, 2018.

[18] H. A. Abd El Aal, L. A. Ahmed, W. A. Hassan, H. M. Fawzy, and H. Moawad, "Combination of carvacrol with simvastatin improves the lipid-lowering efficacy and alleviates simvastatin side effects," Journal of Biochemical and Molecular Toxicology, vol. 31, no. 12, article e21981, pp. 21981-21988, 2017.

[19] A. Ackermann, A. C. Karagoz, A. Ghoochani et al., "Cytotoxic profiling of artesunic and betulinic acids and their synthetic hybrid compound on neurons and gliomas," Oncotarget, vol. 8, no. 37, pp. 61457-61474, 2017.

[20] Y. Sun, L. Sun, S. Liu, J. Song, J. Cheng, and J. Liu, "Effect of emodin on aquaporin 5 expression in rats with sepsisinduced acute lung injury," Journal of Traditional Chinese Medicine, vol. 35, no. 6, pp. 679-684, 2015.

[21] Q. L. Wang, L. Yang, Y. Peng et al., “Ginsenoside Rg1 regulates SIRT1 to ameliorate sepsis-induced lung inflammation and injury via inhibiting endoplasmic reticulum stress and inflammation," Mediators of Inflammation, vol. 2019, Article ID 6453296, 10 pages, 2019.

[22] H. Zhao, M. Zhao, Y. Wang, F. Li, and Z. Zhang, "Glycyrrhizic acid prevents sepsis-induced acute lung injury and mortality in rats," Journal of Histochemistry \& Cytochemistry, vol. 64, no. 2, pp. 125-137, 2015.

[23] X. Shi, G. Chen, J. Wei et al., "UHPLC-Q-TOF MS-based metabolic analysis for the therapeutic efficacy of "Xuebijing injection" against sepsis-induced acute lung injury," EvidenceBased Complementary and Alternative Medicine, vol. 2018, Article ID 8514619, 12 pages, 2018.

[24] R. Yan, Y. Wang, Y. Liu, and X. Di, “Comparative pharmacokinetics of berberine, palmatine and jatrorrhizine in rat plasma after oral administration of Rhizoma coptidis and Zuojinwan using liquid chromatography-tandem mass spectrometry," Iranian Journal of Pharmaceutical Research, vol. 11, no. 3, pp. 949-957, 2012.

[25] P. Qian, Y. B. Zhang, Y. F. Yang, W. Xu, and X. W. Yang, "Pharmacokinetics studies of 12 alkaloids in rat plasma after oral administration of Zuojin and Fan-Zuojin formulas," Molecules, vol. 22, no. 2, pp. 214-231, 2017.

[26] K. He, S. Kou, Z. Zou et al., "Hypolipidemic effects of alkaloids from Rhizoma coptidis in diet-induced hyperlipidemic hamsters," Planta Medica, vol. 82, no. 8, pp. 690-697, 2016.

[27] Y. Lv, J. Wang, D. Xu et al., "Comparative study of single/combination use of Huang-Lian-Jie-Du decoction and berberine on their protection on sepsis induced acute liver injury by
NMR metabolic profiling," Journal of Pharmaceutical and Biomedical Analysis, vol. 145, no. 1, pp. 794-804, 2017.

[28] D. Xu, Y. Lv, J. Wang, M. Yang, and L. Kong, “Deciphering the mechanism of Huang-Lian-Jie-Du-decoction on the treatment of sepsis by formula decomposition and metabolomics: enhancement of cholinergic pathways and inhibition of HMGB-1/TLR4/NF- $\kappa$ B signaling," Pharmacological Research, vol. 121, no. 1, pp. 94-113, 2017.

[29] X. Fan, J. Y. Zhu, Y. Sun et al., "Evodiamine inhibits zymosaninduced inflammation in vitro and in vivo: inactivation of NF$\kappa \mathrm{B}$ by inhibiting $\mathrm{I} \kappa \mathrm{B} \alpha$ phosphorylation," Inflammation, vol. 40, no. 3, pp. 1012-1027, 2017.

[30] M. G. Toscano, D. Ganea, and A. M. Gamero, "Cecal ligation puncture procedure," Journal of Visualized Experiments, vol. 7, no. 51, pp. 2860-2864, 2011.

[31] I. I. Siempos, H. C. Lam, Y. Ding, M. E. Choi, A. M. Choi, and S. W. Ryter, "Cecal ligation and puncture-induced sepsis as a model to study autophagy in mice," Journal of Visualized Experiments, no. 84, article e51066, 2014.

[32] D. Rittirsch, M. S. Huber-Lang, M. A. Flierl, and P. A. Ward, "Immunodesign of experimental sepsis by cecal ligation and puncture," Nature Protocols, vol. 4, no. 1, pp. 31-36, 2009.

[33] L. Dejager, I. Pinheiro, E. Dejonckheere, and C. Libert, "Cecal ligation and puncture: the gold standard model for polymicrobial sepsis?," Trends in Microbiology, vol. 19, no. 4, pp. 198208, 2011.

[34] Q. S. Wang, Y. L. Cui, T. J. Dong, X. F. Zhang, and K. M. Lin, "Ethanol extract from a Chinese herbal formula, "Zuojin Pill", inhibit the expression of inflammatory mediators in lipopolysaccharide-stimulated RAW 264.7 mouse macrophages," Journal of Ethnopharmacology, vol. 141, no. 1, pp. 377-385, 2012.

[35] S. Serafini, M. M. Santos, A. C. Aoun Tannuri et al., "Is hematoxylin-eosin staining in rectal mucosal and submucosal biopsies still useful for the diagnosis of Hirschsprung disease?," Diagnostic Pathology, vol. 12, no. 1, pp. 84-88, 2017.

[36] H. Liu, R. Zhu, C. Liu et al., "Evaluation of decalcification techniques for rat femurs using $\mathrm{HE}$ and immunohistochemical staining," Biomed Research International, vol. 2017, Article ID 9050754, 6 pages, 2017.

[37] A. Sharma, W. L. Yang, M. Ochani, and P. Wang, "Mitigation of sepsis-induced inflammatory responses and organ injury through targeting Wnt/ $\beta$-catenin signaling," Scientific Reports, vol. 7, no. 1, pp. 9235-9248, 2017.

[38] J. Liao, T. Xu, J. X. Zheng et al., "Nitidine chloride inhibits hepatocellular carcinoma cell growth in vivo through the suppression of the JAK1/STAT3 signaling pathway," International Journal of Molecular Medicine, vol. 32, no. 1, pp. 7984, 2013.

[39] H. Li, C. Feng, C. Fan et al., "Intervention of oncostatin Mdriven mucosal inflammation by berberine exerts therapeutic property in chronic ulcerative colitis," Cell Death and Disease, vol. 11 , no. 4, p. 271, 2020.

[40] L. Lou, J. Zhou, Y. Liu et al., "Chlorogenic acid induces apoptosis to inhibit inflammatory proliferation of IL-6-induced fibroblast-like synoviocytes through modulating the activation of JAK/STAT and NF- $\kappa$ B signaling pathways," Experimental and Therapeutic Medicine, vol. 11, no. 5, pp. 2054-2060, 2016.

[41] L. Lou, Y. Liu, J. Zhou et al., "Chlorogenic acid and luteolin synergistically inhibit the proliferation of interleukin- $1 \beta$ induced fibroblast-like synoviocytes through regulating the 
activation of NF- $\kappa \mathrm{B}$ and JAK/STAT-signaling pathways," Immunopharmacology and Immunotoxicology, vol. 37, no. 6, pp. 499-507, 2015.

[42] L. Zhiling, Y. Mingshi, P. Yue, G. Min, and Y. Bingchang, "Rutaecarpine ameliorated sepsis-induced peritoneal resident macrophages apoptosis and inflammation responses," Life Sciences, vol. 228, pp. 11-20, 2019.

[43] Q. Zhang, X. L. Piao, X. S. Piao, T. Lu, D. Wang, and S. W. Kim, "Preventive effect of Coptis chinensis and berberine on intestinal injury in rats challenged with lipopolysaccharides," Food and Chemical Toxicology, vol. 49, no. 1, pp. 61-69, 2011.

[44] J. E. Lee, A. S. Lee, D. H. Kim et al., "Janex-1, a JAK3 inhibitor, ameliorates tumor necrosis factor- $\alpha$-induced expression of cell adhesion molecules and improves myocardial vascular permeability in endotoxemic mice," International Journal of Molecular Medicine, vol. 29, no. 5, pp. 864-870, 2012.

[45] Y. K. Chen, Y. K. Xu, H. Zhang et al., "Emodin alleviates jejunum injury in rats with sepsis by inhibiting inflammation response," Biomedicine \& Pharmacotherapy, vol. 84, pp. 1001-1007, 2016.

[46] C. H. Lang, L. Hong-Brown, and R. A. Frost, "Cytokine inhibition of JAK-STAT signaling: a new mechanism of growth hormone resistance," Pediatric Nephrology, vol. 20, no. 3, pp. 306-312, 2005. 